\title{
LONE PULMONARY HYPERTENSION
}

\author{
By D. S. SHORT, M.D., M.R.C.P.* \\ The Department of Cardiology and the Institute of Clinical Research and Experimental Medicine, The Middlesex Hospital, \\ and the Cardiac Department, The London Hospital
}

Lone (or primary), pulmonary hypertension (P.H.T.) may be defined as a condition of high pressure in the pulmonary artery associated with right ventricular hypertrophy for which no cause can be found outside the pulmonary vessels. The diagnosis is one which is made by exclusion of other diseases which are known to be potential or predisposing causes of P.H.T. The most important of these are chronic lung disease, mitral stenosis and congenital heart disease (Bedford, 1951). Less frequent causes are recurrent pulmonary embolism and pulmonary arteritis.

The forms of lung disease most frequently associated with P.H.T. are emphysema and pneumoconiosis. Fibroid tuberculosis, cystic disease, sarcoidosis, secondary carcinomatosis and primary haemosiderosis are sometimes responsible. Kyphoscoliosis is another rare cause. The most important varieties of congenital heart disease are those which permit a left to right shunt, notably atrial septal defect, ventricular septal defect and patent ductus arteriosus. Repeated pulmonary embolism secondary to thrombosis in the systemic veins is an important and not very rare cause of P.H.T.; so also, in Egypt, is bilharzial pulmonary endarteritis; but rheumatic and syphilitic pulmonary arteritis are very uncommon.

Under the diagnosis of primary pulmonary arteriosclerosis, occasional cases of Lone P.H.T. have been reported since the middle of the last century, but by 1935 Brenner could find reports of only i 5 pure and well authenticated cases. During the past 20 years, and particularly since the introduction of cardiac catheterization, many more cases have been described. The disease is nevertheless a rare one and there are still less than roo adequately reported cases with necropsy control in the world's literature. Dr. William Evans and Dr. Evan Bedford are about to publish a considerable series of cases which were carefully studied both during life and at necropsy. Through their courtesy the present author has had the opportunity of examining several of the patients during

\footnotetext{
* Holding a Leverhulme Scholarship.
}

life, as well as all the case records and postmortem material.

\section{Aetiology}

Symptoms usually make their appearance in the third or fourth decade, less commonly in childhood, though cases have been reported as young as six months and as old as 74 years. The sex incidence is noteworthy, for females outnumber males by approximately two to one. The possibility of a hereditary influence in some cases is suggested by the report of Dresdale et al. (1954) of four probable cases in two generations of one family.

\section{Pathology}

The liver, spleen, kidneys and other systemic organs are usually the seat of chronic venous congestion and occasionally of infarction, but the most important changes are to be found in the heart and lungs.

The heart is moderately enlarged. Its weight is usually about one and a half times the normal and seldom exceeds $55^{\circ} \mathrm{g}$. in the absence of pulmonary incompetence. The striking feature is the enormous hypertrophy of the right ventricle, the thickness of whose wall approaches that of the left ventricle and may even exceed it. There is usually dilatation and hypertrophy of the right atrium, but the leftsided chambers are normal or small.

The lung parenchyma usually appears healthy on naked eye examination, though infarcts are sometimes present and occasionally there is congestion and even oedema. The pulmonary artery trunk and its branches are dilated and intimal thickening is more widespread than usual considering the age of the subject. The segmental arteries (diameter 2 to $5 \mathrm{~mm}$.) often stand out prominently from the cut surface of the lung.

The most significant abnormalities are found in arteries with a diameter less than I $\mathrm{mm}$. Microscopic examination generally reveals widespread intimal thickening, causing obstruction or even occlusion of the small arteries or arterioles. The 
post-mortem pulmonary arteriogram (Evans, I95I ; Short, 1956) is always grossly abnormal and shows that, at least in the final stages, the arterial bed is greatly restricted by structural disease. In addition, there are abnormal anastomoses. Some of these are between adjacent pulmonary arteries, while others link pulmonary and bronchial arteries.

The ultimate cause of Lone P.H.T. is still unknown. Indeed, there is probably more than one disease included under this diagnosis. Many authorities believe that the changes which are found in the arteries at necropsy are a result of the P.H.T., but recent evidence indicates that the arterial disease is generally sufficiently extensive to be regarded as the primary lesion. The nature of the arterial disease remains uncertain. Some of the changes are undoubtedly thrombo-embolic, while others may be secondary to congenital or acquired lesions in the muscular coat.

\section{Symptoms}

The clinical picture of Lone P.H.T. is that of right heart failure appearing suddenly, most frequently in the third or fourth decade, and progressing relentlessly to a fatal termination some two years later.

The first symptom to appear, and the one which dominates the picture throughout, is dyspnoea on effort. A typical story is of a woman finding she could no longer play five sets of tennis. Six months later there were signs of systemic venous engorgement and less than three years from the onset she was dead.

Even when exertion is greatly limited, the patient is usually able to lie flat, but occasionally, particularly in the later stages, there may be orthopnoea or paroxysmal dyspnoea. The explanation of these symptoms is obscure, for pulmonary congestion is rare. Attacks of breathlessness may sometimes be due to pulmonary embolism.

Although less prominent than dyspnoea, pain in the chest on effort is experienced at some time by the majority of patients with Lone P.H.T. It is usually described as a tightness or constriction across the chest, which may radiate into the throat and arms. Except for the fact that it is always associated with dyspnoea, pulmonary angina is indistinguishable from the pain of coronary artery disease. Indeed, it probably has a similar origin, though in P.H.T. it is the right ventricle rather than the left which suffers from ischaemia, since the extra demands of its hypertrophied fibres cannot be met on account of the low and fixed cardiac output.

Other varieties of pain in the chest may be encountered, such as a sense of oppression behind the sternum resulting from pulmonary embolism, or pleuritic pain from pulmonary infarction. Pain in the epigastrium on effort may have its origin in the liver; so-called effort hepatalgia.

The only other symptom which demands consideration is syncope, a history of which is found in about half the patients with Lone P.H.T. Syncope may occur either on effort or at rest. Effort syncope is due to acute right heart failure. During, or shortly after, exercise the cardiac output in these patients falls until it reaches a level where compensatory constriction is unable to maintain the systemic arterial pressure above the critical level of $50 \mathrm{~mm}$. Hg. (Howarth and Lowe, 1953). The exhaustion which these patients experience on slight exertion is probably due to an inadequate blood supply to the voluntary muscle resulting from the same mechanism.

The reason why patients with P.H.T. faint at rest is less certain. Their blood pressure is frequently low and it may be that this renders them abnormally liable to the common vaso-vagal syncope. Sometimes attacks of unconsciousness antedate dyspnoea and cause confusion with idiopathic epilepsy.

\section{Physical Signs}

On examination cyanosis is often evident, but it is rarely profound and may even be absent. The cyanosis is mainly peripheral, due to the low cardiac output, so that it is most apparent on exposed parts, such as the lips, cheeks, ears and hands. It is practically never accompanied by definite clubbing, except in children. Where cyanosis is doubtful a short walk usually makes it obvious.

Signs of systemic venous congestion make their appearance sooner or later in most cases. The earliest indication of this stage is engorgement of the jugular veins. Long before the pressure in the great veins begins to rise, however, there is a remarkable change in the form of the venous pulse. The auricular wave becomes exaggerated and in extreme cases may rise as much as $10 \mathrm{~cm}$. above the diastolic level. Because of its quick rise and fall, this wave may be mistaken for the carotid pulsation of aortic incompetence unless it is carefully timed. With the onset of congestive failure the auricular wave becomes less prominent and may ultimately be overshadowed by the systolic wave of tricuspid incompetence.

The radial pulse in P.H.T. is almost invariably small on account of the low cardiac output and the extremities are cold. The blood pressure is usually low, but peripheral vaso-constriction may sometimes maintain a diastolic pressure of I IO mm. $\mathrm{Hg}$. or higher.

Palpation provides evidence of right ventricular enlargement. The cardiac impulse is as a rule 


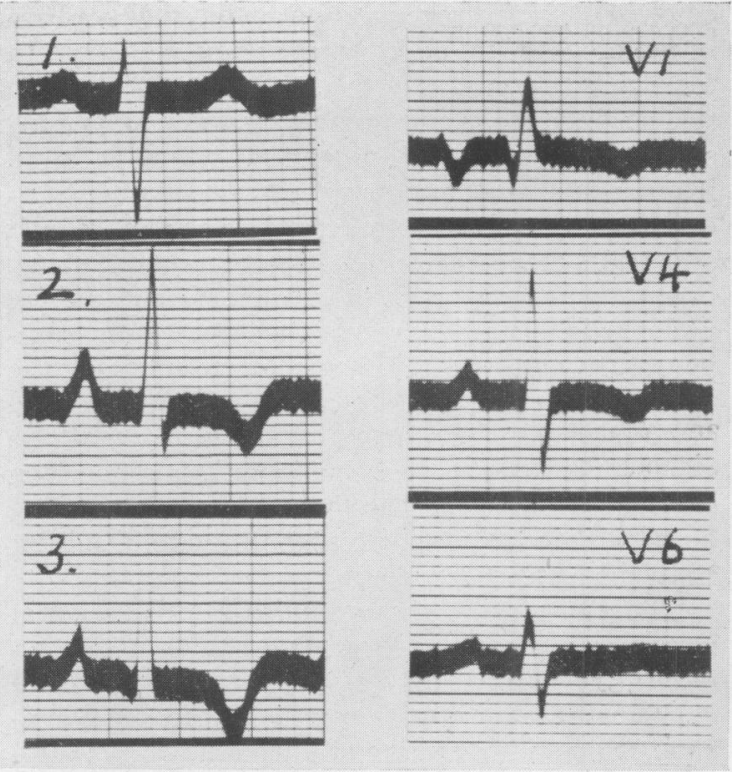

FIG. 1.-The electrocardiogram from a case of Lone P.H.T. showing the pattern of gross right heart hypertrophy.

diffuse, and pulsation extends medially almost to the sternum and upward to the third left intercostal space. Palpation in the second space may reveal abnormal pulsation of the pulmonary artery and often an abrupt click synchronous with pulmonary valve closure.

On auscultation the second sound is usually the most striking event in the cardiac cycle and is well heard all over the precordium. At the pulmonary area splitting of this sound is usually obvious, though not abnormally wide, and the second element, indicating pulmonary valve closure, is much louder than the first or aortic component. Triple heart rhythm is generally present and may be due either to a loud auricular sound or to an audible third sound.

Other auscultatory findings are variable. In the pulmonary area there may be an abrupt sound or click in early systole, and this may be followed or replaced by an ejection murmur. The most frequent site for a systolic murmur is the apex, less commonly the third or fourth left intercostal space.

A diastolic murmur is heard in almost half the cases of Lone P.H.T. Most commonly it is the blowing murmur of pulmonary incompetence, which immediately follows the second heart sound, and is best heard at the point where the intensity of this sound is greatest. Rarely a delayed diastolic murmur has been heard.

\section{Investigations}

A slight polycythaemia is usual and haemoglobin

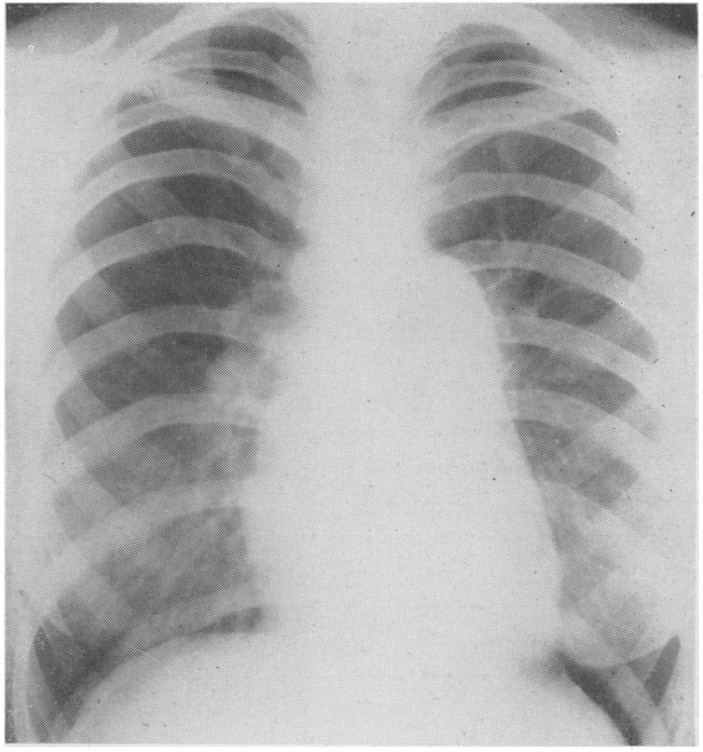

FIG. 2.-The chest X-ray from a case of Lone P.H.T. showing great enlargement of the pulmonary artery, slight enlargement of the right ventricle, and somewhat ischaemic lung fields.

values as high as 140 per cent. with red cell counts of 8 million per c.ml. have been recorded in somes cases. The arterial oxygen saturation is usually reduced to between 80 and 90 per cent. and may occasionally be as low as 70 per cent. The cause of the arterial anoxaemia is not fully understood: A patent foramen ovale is present in some cases and has been held to account for intermittent cyanosis (Edwards et al., 1953). A shunt from the pulmonary to the bronchial circulation through abnormal broncho-pulmonary anastomoses may be the explanation in other cases.

Another unexplained finding is the discovery in nearly half the cases of a polymorphonuclear leucocytosis. Counts of 17,000 cells per c.mm. are frequently observed in the absence of overt pulmonary embolism or infarction.

The electrocardiogram (Fig. I) shows the pattern of right heart hypertrophy, namely, right axis deviation, a dominant $\mathrm{R}$ deflection in the rightsided chest leads $\left(V_{I}, 2\right)$, a prominent $S$ wave in the left-sided leads $(\mathrm{V} 6,7)$, and tall pointed $P$ waves $(P$. pulmonale). Sinus rhythm is invariable.

On $X$-ray examination (Fig. 2 ) the most striking finding is the dilatation of the pulmonary artery trunk, which is sometimes aneurysmal. There is usually moderate enlargement of the right ventricle and right auricle, while the left-sided chambers are small. The general appearance is reminiscent of atrial septal defect and the similarity is increased by the presence of a small aorta in both conditions. 
In Lone P.H.T., however, the right and left pulmonary arteries, though enlarged, are not as pulsatile as in atrial septal defect, and the lung vascularity is never increased and generally much reduced. There may be evidence of old or recent pulmonary infarction (Short, I95I).

The crucial investigation is cardiac catheterization. By this means it is possible, not only to measure the pulmonary artery pressure and the pulmonary arteriolar resistance, but also in most cases to exclude the presence of congenital heart disease and mitral stenosis. In Lone P.H.T. the pulmonary systolic pressure at rest generally lies between 70 and $100 \mathrm{~mm}$. Hg., though much higher pressures have been recorded. The cardiac output is usually reduced to about $2.51 / \mathrm{min}$., i.e. half the normal figure.

Angiocardiography may be useful in excluding thrombo-embolic obstruction of the pulmonary trunk or its main branches, but it is not yet possible to demonstrate obstruction of the smallest arteries and the arterioles during life.

\section{Differential Diagnosis}

The diagnosis of Lone P.H.T. is made by excluding all other causes of P.H.T. The presence of chronic lung disease is suggested by a history of recurrent winter cough and bronchitis or longstanding asthma; by the presence of deep cyanosis, particularly when this is accompanied by obvious clubbing; and by X-ray evidence of structural lung disease. The arterial oxygen saturation is lower and the carbon dioxide saturation higher than in Lone P.H.T. (Platts and Whitaker, 1954).

When mitral stenosis is complicated by severe P.H.T. the differentiation from Lone P.H.T. may cause the greatest difficulty, because in such a case the characteristic diastolic murmur may be absent. Fortunately, the opening snap and the accentuation of the first heart sound are usually retained. X-ray examination may show slight left auricular enlargement or calcification of the mitral valve, but it is sometimes necessary to resort to cardiac catheterization. Occasionally the opposite difficulty arises and a case of Lone P.H.T. is mistaken for mitral stenosis because the third heart sound stimulates an opening snap and rarely because of the presence of a delayed diastolic murmur.

Congenital heart disease can only be excluded with confidence after cardiac catheterization. Its presence should, however, be suspected when the history of disability, cyanosis or a cardiac murmur dates from childhood or infancy.

The other important condition which must be considered before making a diagnosis of Lone P.H.T. is recurrent pulmonary embolism. Recog- nition of this disease is not difficult if there is a clear-cut history of repeated attacks of dyspnoea and evidence of long-standing venous thrombosis, but it may be impossible when this evidence is lacking. Recurrent pulmonary embolism tends to be found in an older age group than Lone P.H.T. and seldom shows such high-grade right ventricular hypertrophy in the electrocardiogram (Lenegre and Gerbaux, 1952).

The diagnosis may be suggested by the chest $\mathrm{X}$-ray, particularly if this shows zones of hypertranslucency or thread-like branches arising from an abnormally dense parent artery. In some cases angiocardiography may be necessary. Emboli tend to obstruct the largest pulmonary arteries and even the pulmonary trunk itself, so that filling defects are usually obvious.

Pulmonary embolism (or thrombosis) is a common complication of pre-existing P.H.T. of any aetiology, so that it may be impossible even at necropsy to be certain whether thrombo-embolism was the cause or the result of the P.H.T.

\section{Prognosis}

The majority of patients with Lone P.H.T. are dead within two years of the onset of symptoms, though survival for as long as I I years is known. Pregnancy leads to rapid deterioration.

\section{Treatment}

Various drugs, such as priscol, veriloid, khellin and the methonium compounds, have been shown to lower the pulmonary arterial pressure, but all have proved ineffective in practice. Stellate ganglion block has also been performed, but without success. If structural changes in the arterial bed are already present when symptoms first appear, the failure of these measures is not surprising.

\section{Acknowledgments}

I am greatly indebted to Dr. Evan Bedford, Dr. William Evans and Dr. Walter Somerville for their help in the preparation of this paper.

\section{BIBLIOGRAPHY}

BEDFORD, D. E. (1951), Proc. Roy. Soc. Med., 44, 597.

BRENNER, O. (1935), Arch. intern. Med., 56, 976.

DRESDALE, D. T. MICHTOM, R. J., and SCHULTZ, M. (1954), Bull. N.YY. Acad. Med., 30, r95.

EDWARDS, J. E., HELMHOLZ, H. F., DuSHANE, J. W., and BURCHELL, H. B. (1953), Proc. 'Staff Meet. Mayo Clin., 28, 113 .

EVANS, W. (1951), Proc. Roy. Soc. Med., 44, 600.

HOWARTH, S., and LOWE, J. B. (1953), Brit. Heart F., 15, 47.

LENEGRE, J., and GERBAUX, A. (1952), Arch. Mal. Coeur, 45, 289 .

PLATTS, M. M., and WHITAKER, W. (1954), Amer. Heart $\mathcal{F}$., $48,77$.

SHORT, D. S. (1951), Quart. F. Med., 20, 233.

SHORT, D. S. (1956), Ұ. Fac. Radiol., to be published. 